\title{
Bilrost: Connecting the Internet of Things through human Social Networks with a Domain-Specific Language
}

\author{
Daniel Meana-Llorián; Cristian González García; \\ Jordán Pascual Espada \\ MDE-RG, Department of Computer Science \\ University of Oviedo \\ Oviedo, Spain \\ danielmeanallorian@gmail.com; \\ gonzalezgarciacristian@hotmail.com; \\ pascualjordan@uniovi.es
}

\author{
Manju Khari \\ Department of Computer Science \\ AIACT\&R \\ Geeta Colony, Delhi- 110031 \\ manjukhari@yahoo.co.in
}

\author{
Vijay Bhaskar Semwal \\ Department of Robotics \& Artificial Intelligence \\ Indian Institute of Information Technology \\ Allahabad, India \\ vsemwal@gmail.com
}

\begin{abstract}
Nowadays, we have many Smart Objects near us connected to the Internet. These objects could make things together if an easy platform existed. There are many researches about interconnecting Smart Objects but we propose a novel approach using human Social Networks and a Domain-Specific Language. This approach makes easier the creation of intercommunications not only among objects but also between objects and humans. We propose a novel DSL that allows defining objects with sensors and actuators, and defining connections to Social Networks in order to publish the sensors' values and to do actions based on events triggered by messages. Moreover, with the DSL we will be able to establish external actions and status in order to make the objects be aware of the environment.
\end{abstract}

Keywords-Internet of Things; Smart Objects; Model-Driven Engineering; Domain-Specific Language; Social Networks

\section{INTRODUCTION}

Currently, the Internet of Things (IoT) is a term very popular because everybody has Smart Objects in his pocket like smartphones, wearables, tablets [1], and many others devices connected to the Internet. The possibilities of IoT are very diverse and they can include from Smart Homes [2]-[4] to Smart Earth [5], Smart Cities [6]-[8] or any type of intelligence located in heterogeneous and ubiquitous objects.

The interconnection among objects is very important in the context of the IoT. Smart Objects can be located in places separated by millions of kilometres or in unreachable places. Nevertheless, the objects can be connected and take decisions considering other Smart Objects. However, there are many problems related with the interconnections. First, there is not a unique standard or protocol that allow interacting with objects. Moreover, everybody has not knowledge about programming or creating programs that established the connection among objects or even, they have not knowledge about how to interact with objects.

The aim of this proposal is the creation of applications using a Domain-Specific Language (DSL) created applying Model-Driven Engineering (MDE) With these applications, devices with sensors or/and actuators would be connected to other devices through Social Networks. We propose the creation of a language that defines the different required aspects to define the devices, the data required by Social Networks, the sensors with their properties, the actuators with their properties and conditions that make the actuators run or make the devices publish their sensors' values.

Moreover, the usage of Social Networks prevents us from developing a specific platform and it is a solution more intuitive for people who use Social Networks daily.

The remainder of the paper is organised as follows. In Section II, we introduce involved topics in this research the works related. Section III shows our proposal, the Bilrost platform, how Bilrost DSL, called Bilrost-Specific Language (BSL), is. Section IV contains the conclusions of this paper and the possible future work that can be done from here.

\section{STATE OF THE ART}

In order to present a theoretical frame of our proposal, we have to talk about the Internet of Things, Smart Objects, Online Social Networks, Model-Driven Engineering, and the related work. 


\section{A. The Internet of Things}

Currently, the IoT is one of the most important topics in many researches and business [9]-[12]. It could define as the interconnection between heterogeneous and ubiquitous objects between themselves.

\section{B. Smart Objects}

One important part in the IoT are the Smart Objects. They can be classified in three dimensions [13]: Level of Intelligence, Location of Intelligence, and Aggregation level of Intelligence. The first one indicates the object's intelligence. The second one describes if the intelligence belongs to the object or to the network. The last dimension indicates if the intelligence is in the element, for example, when the object is composed of various elements and each one has their own intelligence, or in the container when it is the contrary case.

Our proposal offers Smart Objects with an intelligence in the container. The other two characteristics depend on the implementation that the user made in the devices.

\section{Online Social Networks}

However, according to interconnect different objects, we need some network. They could be WSAN, IoT Networks or, in our case, Online Social Networks (OSNs). OSNs provide many services to create applications like identity and authorization services, Application Programming Interfaces (APIs) to read or write in timelines, receive updates, receive and send private messages, and so on. OSN is a basic piece of the Web 2.0 and the convergence of the real world with OSNs allows the development of new applications which interconnect things and humans [14].

Moreover, scientists of Ericsson [15] observed that if there is an analogy of using IoT technologies and Social Networks, people are capable of familiarising better with IoT technologies.

\section{Model-Driven Engineering}

Model-Driven Engineering (MDE) appeared to solve software development problems that we have had since the 1960s [16]. With MDE we can automate processes to make the creation of repetitive process easier [17]. Applying MDE, developers can abstract the problem and automate some parts to facilitate the production of similar solutions. For instance, with the creation of Domain Specific Languages (DSL).

\section{E. Related Work}

Our proposal is a novel way of intercommunicating Smart Objects using actual human Social Networks instead of common web services to publish messages. These messages could be a sensor's status or calls to actuators' actions. However, there are not very similar researches related to this proposal. Some research that deals with these issues about interconnecting objects through the Internet are based on Service Oriented Architecture (SOA) like REST [18]-[20]. However, we propose the usage of OSNs to establish the communication not only among Smart Objects but also between humans and Smart Objects. Related to the communication among objects there are some researches like [15], [21] and between humans and objects could be considered [22]-[25].

There are some IoT platforms that allow interconnecting objects like Midgar [9], [26]. Midgar uses a graphic DSL to make the creation of the interconnection easier for people without out development skills. However, Midgar has the requirement of use a server and only allows interconnecting objects whereas Bilrost does not need any server because of the usage of OSNs and allows interconnecting objects with objects and humans with objects.

However, Social Access Controller (SAC) [22] could be an approach similar to our proposal. It uses Social networks to share Smart Objects and it allows managing them and knowing their states. However, the usage of the Social Networks is very different. It uses it to share your Smart Objects with your friends in order to allow them to use it.

The interconnection not only can be done through Social Networks. Old researches used instant messages to interconnect objects and humans [23]-[25]. The principal disadvantage of this approach is the dependence on specific applications which were developed to the research whereas Social Networks are used by everybody.

\section{BILROST PlATFORM}

Bilrost platform is our proposal to investigate the possibility of interconnecting Smart Objects through human Social Networks. The usage of human Social Networks gives us the possibility of interconnecting objects in an environment enough tested by millions of real users. Moreover, these networks not only allow us to interconnect objects but also allow us to interconnect humans and objects. In this way, we would be making the Internet of Things bigger due to the addition of people to the network.

We propose the usage of a DSL to make easier the creation of the interconnections. However, in first steps, Bilrost will not be able to implement the logic to access to the sensors' values or to implement the logic to control the devices' actuators. The users will have to implement this logic in the project generated by Bilrost. So, Bilrost will be able to generate projects that will be able to interconnect objects through Social Networks but the users will have to complete them with the specific logic for each device.

Therefore, our proposal will have the two principal parts: projects generation and specific logic programming.

In this section, we will present how the generated projects will be able to interconnect objects through Social Networks and the two stages in which the user interaction will be required, the projects generation and the completion of the project.

\section{A. Interconnecting objects through Social Networks}

The aim of Bilrost will be the interconnection between objects through human Social Networks. Currently, there are many popular Social Networks like Whatsapp, Facebook, Twitter, and many others that we could use in our proposal. 


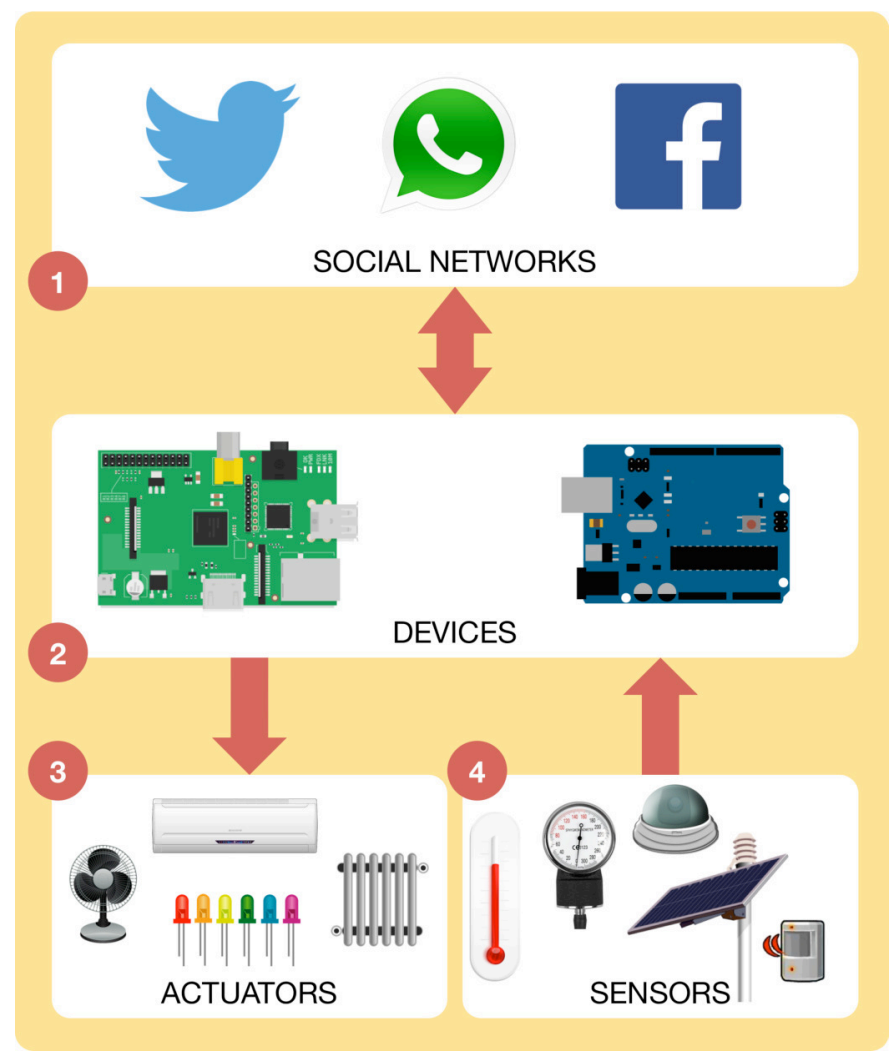

Fig. 1. Interconnection of devices through Social Networks.

However, in the first stages of our proposal, we will use Twitter because of its philosophy of short public messages and the common use of keywords, which are called hashtags.

Bilrost will create projects that devices will be capable of running. For that, we will implement a DSL that we call Bilrost Specific Language (BSL). The content of the BSL will be described in the subsection III.B. After processing the BSL program, Bilrost platform will be able to generate projects where the connection to the Social Networks will have already been implemented.

The generated projects will connect devices like Raspberry Pi or Arduino to the Social Networks selected by the users. These projects will have specific parts that retrieve the sensors' values and do actions with the actuators. As Fig. 1 shows, the devices will be connected to Social Networks in order to publish the retrieve sensors' value and to do the actuators' actions invoked by Social Networks' messages.

In order to explain how Bilrost will work, we will use a simple example. We want to interconnect a Raspberry Pi and an Arduino with several sensors and actuators. Fig. 1 represents these Arduino and Raspberry Pi as devices with the number 2, their sensors with the number 3 and their actuators with the number 4. The Arduino is controlling a door lock and it has a light sensor. The Raspberry Pi has a presence sensor and it controls the lights of a room. With Bilrost, we will be able to interconnect the two devices through a Social Network like Twitter which is represented in Fig. 1 with the number 1. If the Arduino detected that the light level is low, it would publish in Twitter, using special keywords, the level of the light. Then, the Raspberry Pi would receive the level of the light and it would turn on the light if the value sensed accomplish certain conditions. Moreover, if the Raspberry Pi detected a person, it would publish on Twitter that it detected anything and the Arduino would lock the door in order to prevent this person from going into the room.

This example will possible with Bilrost. Using the BSL, we will be able to specify the language of resulting projects, the keywords to use when publish messages or when listen messages, the usernames that the devices can listen, the tokens required by the Social Networks APIs, the actuators and their possible actions that the devices have, the sensors that the devices have and how the devices have to publish the sensors' status like the refreshing time. Furthermore, with the BSL, we will also be able to specify external states and actions. The external states will allow Bilrost to invoke actions of its own actuators when other devices publish a state that accomplishes certain conditions specified with the BSL, and the external actions will allow Bilrost to invoke actuators' actions of other devices when the state of its own sensors accomplish certain conditions also specified with the BSL.

Besides, the usage of human Social Networks will open the possibility of handle human messages like they were messages from other devices. Thus, humans will be able to manage the devices. For example, a person will be able to turn off the heating system remotely publishing a message in a Social Network or a person will be able to know the temperature of its house searching the messages published by the device that has a temperature sensor located at his house.

However, there is a limitation of our proposal. In the early stages, the user will have to implement the access to the sensors' values and the invocation to actuators' actions inside of the projects generated by Bilrost. Bilrost will have the capacity of using users' implementation without any other interaction.

\section{B. The User Interaction}

Bilrost will use a DSL, called BSL, to define devices with actuators and sensors, and also, to define the Social Networks that it will use to connect the devices and the external actions and events that the devices will listen through the Social Network. However, the user interaction is required to define the BSL that generates the projects and to complete the generated projects as Fig. 2 shows. In this section, we will explain these two stages.

\section{1) Projects Generation}

In order to generate a project that connects a device to a Social Network, firstly, the user will have to define the device using BSL. With BSL, the user will be able to define the programming language of the generated project thinking in the device where the project will run. Running the project on a smartphone is not the same than running the project on a Raspberry $\mathrm{Pi}$ because the first one needs programs programmed with Java language and the second one needs programs programmed with Python language. However, due to our proposal, the users do not need advanced programming knowledge in these languages because they only will have to implement the access to the sensors' values and the actuators' 


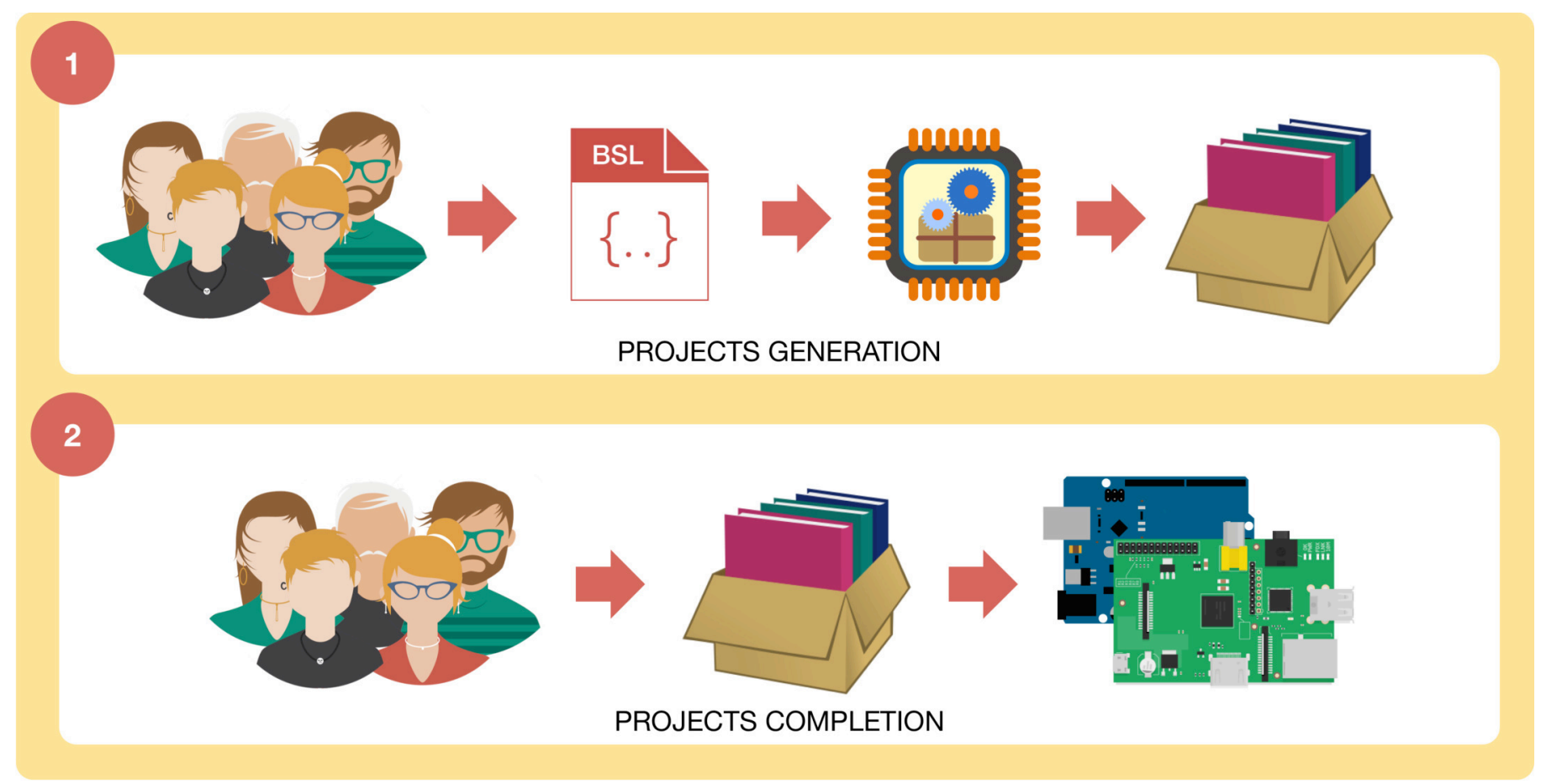

Fig. 2. Stages where there is some user interaction: 1. Projects generation and 2. Projects completion.

actions, they will not implement the connection to Social Networks and many other aspects related to the connection.

Fig. 2 shows the process of projects generation with the number 1 . This stage will start with the users of Bilrost. These users will have to define the BSL as they want. Afterwards, Bilrost will process the BSL file and it will generate a project that accomplishes the rules defined in it the BSL.

The final project will be a project in a specific programming language for the device, and it will contain the logic required to connect the device to the Social Network specified in the BSL with the data required by the Social Network's API. Moreover, the project will have the necessary logic to invoke certain empty methods that users will have to complete, as we will explain in subsection III.B.2, and to accomplish the conditions specified in the BSL related with external states and actions as we explained in section III.A.

\section{2) Projects Completion}

The project generation will not be the last stage in which the user interaction will be required. The users will have to complete the projects that Bilrost will generate with the logic that retrieves the sensors' value and with the logic that controls the actuators. Our proposal does not include the generation of specific code because we are focused on the interconnection of objects through Social Networks. Thus, the specific code will have to be coded by the users, at least in the first stages of the investigation.

Fig. 2 shows the process of projects completion with the number 2. This stage will start with the users of Bilrost. The users will have to take the generated project and update it. The projects will have methods with an empty body but with descriptions that will explain to the users what they will have to implement and how they will use the existing code.
Afterwards, the users will have to deploy the project in the devices and run it.

\section{CONCLUSIONS}

We presented our proposal about interconnecting Smart Objects from the Internet of Things through human Social Networks. The usage of human Social Networks opens the possibility of interconnect objects and humans. Thus, our proposal would make the Internet of Things bigger because of the addition of humans. In order to make the connection easier, we proposed the used of a Domain-Specific Language to define devices that are connected to Social Networks using specific keywords. The Bilrost Specific Language will also define the devices' actuators with their actions and the devices' sensors with the time that it will be used to decide when to publish the sensed value. Moreover, the BSL will define external states that the devices will have to interpret and respond doing some action with their actuators and external actions that the devices will be able to use in order to invoke actuators located in other devices.

However, more work can be done related to this proposal. One possible future work could be the addition of the specific implementation in the BSL like the way of getting the sensors' values or the logic of the actuators' actions. Moreover, Social Networks are not similar so, doing an investigation that analyses the available Social Networks in order to decide the best one to use with objects is a good future work too. Finally, we made our proposal thinking in the Internet of Things and devices like Arduino or Raspberry Pi but there are many others fields where our proposal could be used and a future work is to discover it. 


\section{ACKNOWLEDGMENT}

This work was performed by the "Ingeniería Dirigida por Modelos MDE-RG" research group at the University of Oviedo under Contract No. FC-15-GRUPIN14-084 of the research project "Ingeniería Dirigida Por Modelos MDE-RG". Project financed by PR Proyecto Plan Regional.

\section{REFERENCES}

[1] Fundación Telefónica, La Sociedad de la Información en España 2014. Grupo Planeta Spain, 2015.

[2] H. Gu and D. Wang, "A Content-aware Fridge based on RFID in smart home for home-healthcare," in 2009 11th International Conference on Advanced Communication Technology, 2009, vol. 2, pp. 987-990.

[3] C. Han, J. M. Jornet, E. Fadel, and I. F. Akyildiz, "A cross-layer communication module for the Internet of Things," Comput. Networks, vol. 57, no. 3, pp. 622-633, Feb. 2013.

[4] D. Ding, R. A. Cooper, P. F. Pasquina, and L. Fici-Pasquina, "Sensor technology for smart homes," Maturitas, vol. 69, no. 2, pp. 131-136, Jun. 2011.

[5] D. Li, Y. Yao, Z. Shao, and L. Wang, "From digital Earth to smart Earth," Chinese Sci. Bull., vol. 59, no. 8, pp. 722-733, Mar. 2014.

[6] L. Sanchez et al., "SmartSantander: IoT experimentation over a smart city testbed," Comput. Networks, vol. 61, pp. 217-238, Mar. 2014.

[7] D. Meana-Llorián, C. González García, B. C. Pelayo G-Bustelo, J. M. Cueva Lovelle, and N. Garcia-Fernandez, "IoFClime: The fuzzy logic and the Internet of Things to control indoor temperature regarding the outdoor ambient conditions," Futur. Gener. Comput. Syst., p. , Nov. 2016.

[8] C. González García, D. Meana-Llorián, B. C. Pelayo G-Bustelo, J. M. Cueva Lovelle, and N. Garcia-Fernandez, "Midgar: Detection of people through computer vision in the Internet of Things scenarios to improve the security in Smart Cities, Smart Towns, and Smart Homes," Futur. Gener. Comput. Syst., p. , Jan. 2017.

[9] C. González García, B. C. Pelayo G-Bustelo, J. Pascual Espada, and G. Cueva-Fernandez, "Midgar: Generation of heterogeneous objects interconnecting applications. A Domain Specific Language proposal for Internet of Things scenarios," Comput. Networks, vol. 64, pp. 143-158, May 2014.

[10] L. Atzori, A. Iera, and G. Morabito, "The Internet of Things: A survey," Comput. Networks, vol. 54, no. 15, pp. 2787-2805, Oct. 2010.

[11] K. Gama, L. Touseau, and D. Donsez, "Combining heterogeneous service technologies for building an Internet of Things middleware," Comput. Commun., vol. 35, no. 4, pp. 405-417, Feb. 2012.

[12] Lu Tan and Neng Wang, "Future internet: The Internet of Things," in 2010 3rd International Conference on Advanced Computer Theory and Engineering(ICACTE), 2010, vol. 5, pp. V5-376-V5-380.
[13] C. González García, D. Meana-Llorián, C. Pelayo G-Bustelo, and J. M. Cueva-Lovelle, "A review about Smart Objects, Sensors, and Actuators," Int. J. Interact. Multimed. Artif. Intell., vol. 4, no. 3, p. 7, 2017.

[14] M. Blackstock, R. Lea, and A. Friday, "Uniting online social networks with places and things," in Proceedings of the Second International Workshop on Web of Things, 2011, p. 5:1--5:6.

[15] L. Atzori, A. Iera, and G. Morabito, "From 'smart objects' to 'social objects': The next evolutionary step of the internet of things," IEEE Commun. Mag., vol. 52, no. 1, pp. 97-105, Jan. 2014.

[16] E. Dijkstra, "The humble programmer," Commun. ACM, vol. 15, no. October 1972, pp. 859-866, 1972.

[17] V. García-Díaz, H. Fernández-Fernández, E. Palacios-González, B. C. P. G-Bustelo, O. Sanjuán-Martínez, and J. M. C. Lovelle, "TALISMAN MDE: Mixing MDE principles," J. Syst. Softw., vol. 83, no. 7, pp. 1179 1191,2010

[18] D. Guinard and V. Trifa, "Towards the Web of Things: Web Mashups for Embedded Devices," in Workshop on Mashups, Enterprise Mashups and Lightweight Composition on the Web (MEM 2009), in proceedings of WWW (International World Wide Web Conferences), 2009, pp. 1-8.

[19] D. Guinard, V. Trifa, and E. Wilde, "A resource oriented architecture for the web of things," in 2010 Internet of Things, IoT 2010, 2010, pp. 1-8.

[20] D. Guinard, V. Trifa, S. Karnouskos, P. Spiess, and D. Savio, "Interacting with the SOA-based internet of things: Discovery, query, selection, and on-demand provisioning of web services," IEEE Trans. Serv. Comput., vol. 3, no. 3, pp. 223-235, 2010.

[21] L. Atzori, A. Iera, and G. Morabito, "SIoT: Giving a Social Structure to the Internet of Things," IEEE Commun. Lett., vol. 15, no. 11, pp. 1193 1195, Nov. 2011.

[22] D. Guinard, M. Fischer, and V. Trifa, "Sharing using social networks in a composable Web of Things," in Pervasive Computing and Communications Workshops (PERCOM Workshops), 2010 8th IEEE International Conference on, 2010, pp. 702-707.

[23] J. Choi and C. W. Yoo, "Connect with things through instant messaging," in Lecture Notes in Computer Science (including subseries Lecture Notes in Artificial Intelligence and Lecture Notes in Bioinformatics), vol. 4952 LNCS, Springer, 2008, pp. 276-288.

[24] S. Aurell, "Remote controlling devices using instant messaging: building an intelligent gateway in Erlang/OTP," in Proceedings of the 2005 ACM SIGPLAN workshop on Erlang, 2005, pp. 46-51.

[25] A. Roychowdhury and S. Moyer, "Instant messaging and presence for sip enabled networked appliances," Publ. Unkn., 2001.

[26] C. González García, J. Pascual Espada, E. R. Núñez-Valdez, and V. García-Díaz, "Midgar: Domain-Specific Language to Generate Smart Objects for an Internet of Things Platform," in 2014 Eighth International Conference on Innovative Mobile and Internet Services in Ubiquitous Computing, 2014, pp. 352-357. 